\title{
Reconstruction of esophageal stenosis that had persisted for 40 years using a free jejunal patch graft with virtual endoscopy assistance
}

\author{
Daisuke Fujisawa ${ }^{1}$, Hirotaka Asato ${ }^{1}$, Katsunori Tanaka ${ }^{2}$, Tetsuo Itokazu ${ }^{2}$, Shizuo Kojya $^{2}$ \\ ${ }^{1}$ Department of Plastic and Reconstructive Surgery, Dokkyo Medical University, Tochigi; ${ }^{2}$ Department of Otorhinolaryngology, Head and \\ Neck Surgery, Nakagami Hospital, Okinawa, Japan
}

In this report, we present a case in which good results were achieved by treatment using a free jejunal patch graft with virtual endoscopy (VE) assistance in a patient whose swallowing had failed to improve for 40 years after he mistakenly swallowed sulfuric acid, despite pectoralis major myocutaneous flap grafting and frequent balloon dilatation surgery. During the last 20 years, virtual computed tomography imaging has improved remarkably and continues to be used to address new challenges. For reconstructive surgeons, the greatest advantage of $V E$ is that it is a noninvasive modality capable of visualizing areas inaccessible to a flexible endoscope. Using VE findings, we were able to visualize the 3-dimensional shape beyond the stenosis. VE can also help predict the area of the defect after contracture release.

Keywords Virtual endoscopy / Esophageal stenosis / Burn, chemical / Jejunal transfer
Correspondence: Daisuke Fujisawa Department of Plastic and Reconstructive Surgery, Dokkyo Medical University, 880 Kitakobayashi, Mibu, Shimotsuga, Tochigi 321-0293, Japan

Tel: $+81-282-86-1111$

Fax: +81-282-86-1806

E-mail: dai-fuji@dokkyomed.ac.jp

This article was presented at the 45th Annual Meeting of the Japanese Society for Reconstructive Microsurgery, on December 6-7, 2018, in Osaka, Japan.

Received: January 22, 2019 • Revised: February 22, $2019 \bullet$ Accepted: March 6, 2019

pISSN: 2234-6163 • elSSN: 2234-6171 • https://doi.org/10.5999/aps.2019.00101 • Arch Plast Surg 2020;47:178-181

\section{INTRODUCTION}

During the last 20 years, virtual computed tomography (CT) imaging has improved remarkably and continues to be used to address new challenges. Vining et al. [1] first reported the relationships of virtual endobronchial simulations to actual bronchoscopic findings. Initially, this virtual endoscopy (VE) technique was considered to be useful for prebronchoscopy planning, endoscopy training, and/or endobronchial therapy and research. The usefulness of VE for esophageal atresia and tracheal stenosis in neonates, infants, and children was reported [2]. Gradually, VE has entered into use not only for the identification of stenotic sites, but also for the diagnosis of various esophageal pathologic conditions [3], and its use for staging $\mathrm{T}$ and $\mathrm{N}$ parameters of esophageal cancer has been evaluated [4].

Since the report of Seidenberg et al. [5], pharyngoesophageal defects have been reconstructed using jejunal transfer, especially in patients in whom a gastric tube cannot be placed. Free jejunal transfer is the preferred option for hypopharyngeal or esophageal defects. For reconstruction after partial hypopharyngectomy with laryngeal preservation, the use of a free jejunal patch graft (FJPG) [6] has been widely accepted as the method of first choice $[7,8]$. In this report, we present a case in which we obtained good results by using an FJPG with VE assistance to treat a patient whose swallowing had failed to improve for 40 years after he mistakenly swallowed sulfuric acid, despite pectoralis major myocutaneous (PMMC) flap grafting and frequent balloon dilatation surgery. 


\section{CASE}

Our patient was a 70-year-old man with severe dysphagia as a result of accidental ingestion of sulfuric acid while intoxicated when he was roughly 30 years old. He was initially treated by subtotal esophagectomy with gastric tube-cervical esophageal anastomosis. Severe dysphagia continued afterwards, and in 2005 (at the age of 58 years) he visited the Department of Surgery of Nakagami Hospital. Despite undergoing endoscopic esophageal dilatation several times every year, his symptoms did not improve, and he underwent contracture release and PMMC flap transfer in 2009 (at the age of 62 years). He also underwent periodic endoscopic esophageal dilatation thereafter (up to five times per year). He was referred from the department of surgery to the department of plastic surgery in May 2016. On examination, there was a surgical scar on his neck, and a scar where the right PMMC flap had been harvested. There was also a scar in the center of his abdomen from the first operation, as shown in Fig. 1. He complained of extreme difficulty in swallowing, leading him to squeeze his neck manually to pass food. Endoscopic examination by an otolaryngologist showed no major problems in his larynx, and it was considered that the food that he consumed entered the gastric tube via the PMMC flap from the right piriform sinus. It was impossible to advance the endoscope

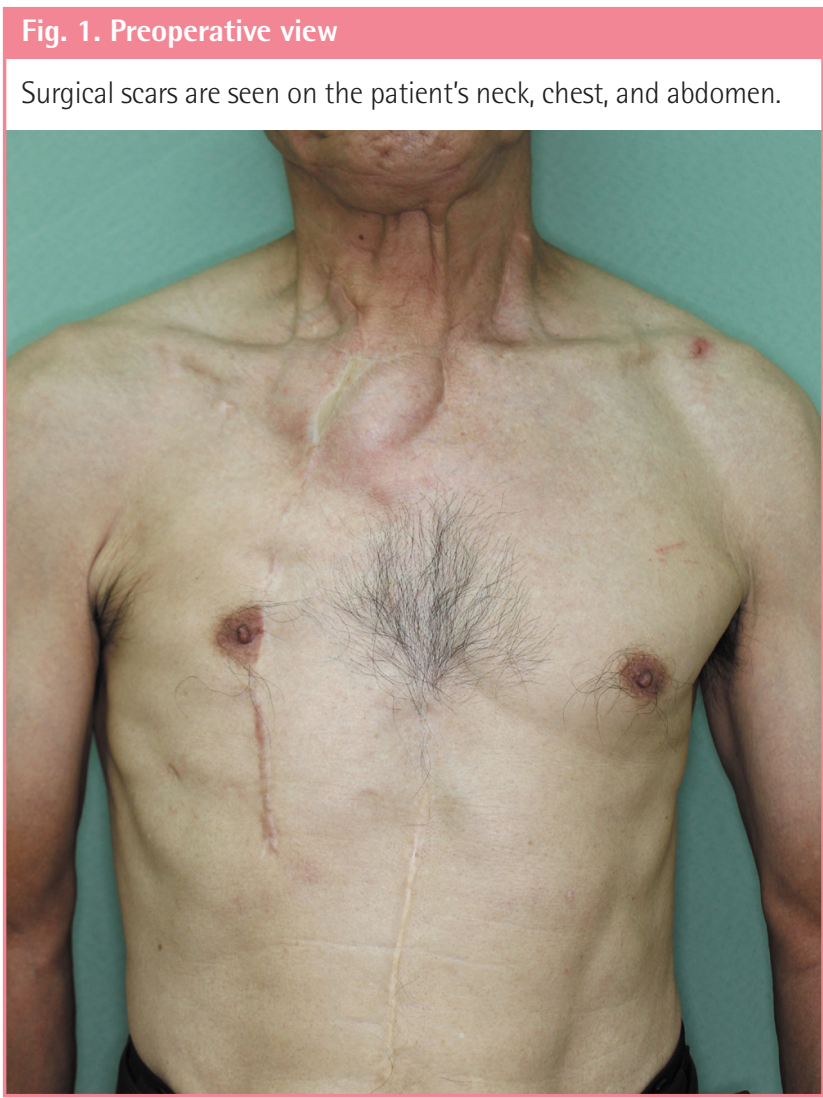

forward due to the severe stenosis. Three-dimensional multidetector CT (MDCT) and VE were performed. MDCT was performed using a 64-slice CT apparatus (Aquilion 64; Toshiba Medical Systems Corp., Otawara, Japan). The technical parameters were $120 \mathrm{kVp}, 150 \mathrm{~mA}$, collimation of $64 \times 0.5$, a rotation time of 0.5 , a slice thickness of $1 \mathrm{~mm}$, and an increment of 1 $\mathrm{mm}$. On VE, the stenosis was about $2-3 \mathrm{~cm}$ in length. A contrast swallowing examination also showed a similar result, as shown in Fig. 2. Based on the VE findings, we decided to utilize a patch graft, for which we expected that the luminal mucosa could be used to some extent. Thus, surgery was planned to release the stenosis and replace it with a FJPG.

In August 2016, a joint operation took place. A U-shaped skin incision was made in the neck. The transplanted right PMMC flap was dissected. Using a nerve-stimulating device, peeling of the flap and esophageal stricture was performed. Ultimately, the PMMC flap was excised, and the leftover epithelium remained in the lumen. As shown in the preoperative VE image of the right piriform sinus, pinhole-like stenosis was observed.

The stenosis was incised and expanded sufficiently with fin-

\section{Fig. 2. Preoperative VE and swallowing images}

(A, B) Virtual endoscopy (VE) findings from the oral side (white asterisk: right piriform sinus). (C, D) VE findings from the inferior side. (E) Findings from the contrast swallowing examination.
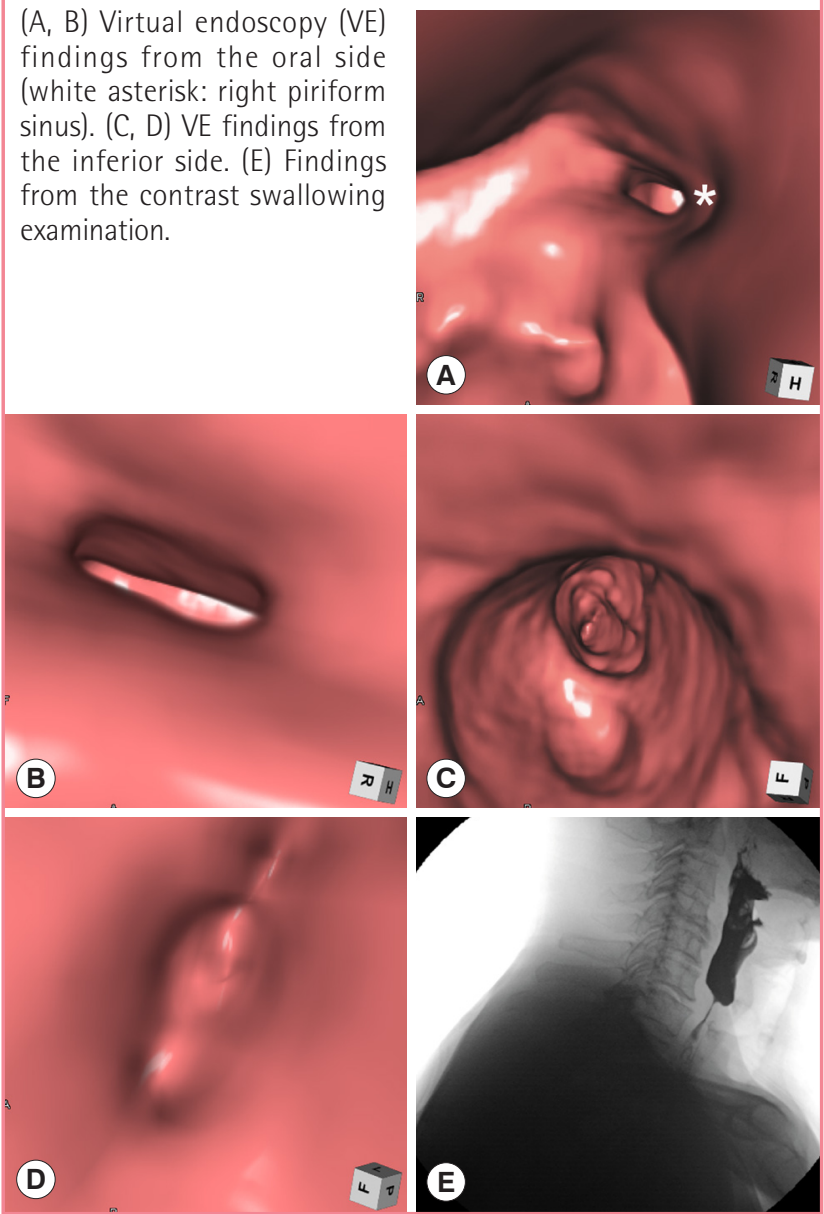
Fig. 3. Postoperative swallowing image and endoscopy

(A) The contrast swallowing examination performed at 3 months postoperatively confirmed good passage. (B) Findings of upper gastrointestinal endoscopy at 2 years and 3 months postoperatively. No esophageal stricture was seen (black asterisk: patch graft).
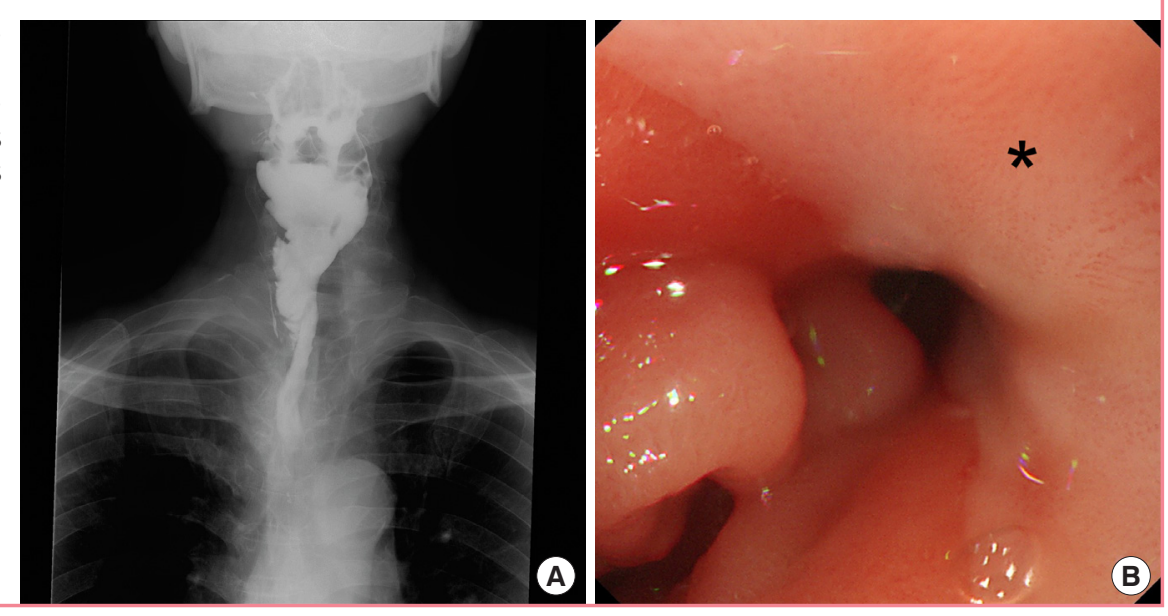

gers on both the cephalad and caudal sides. The right piriform sinus remained only on the left wall (inner wall), and it was decided to reconstruct the three sides of the front, the rear, and the outer walls with a FJPG. The defect length was about $5 \mathrm{~cm}$, in a spindle shape. The harvested jejunum was split in the longitudinal direction, and was divided into a patch graft and a monitoring segment. The FJPG was transferred in a $90^{\circ}$-transposed fashion. The third jejunal artery and the right transverse cervical artery were end-to-end anastomosed with 9-0 nylon sutures. The third jejunal vein and the right internal jugular vein were end-to-side anastomosed with 9-0 nylon sutures.

The flap survived completely. Videofluorography was performed at 3 months postoperatively, and good passage was confirmed, as well as at 2 years postoperatively. There were no complications, such as an abdominal wall hernia. The patient was able to enjoy meals and his body weight increased by $10 \mathrm{~kg}$. Upper gastrointestinal endoscopy was performed at 2 years and 3 months after surgery. Superficial gastritis was found in only a part of the gastric tube, and no evidence of stricture or cancer was seen, as shown in Fig. 3.

\section{DISCUSSION}

As a means of evaluating mucosa in the acute phase of chemical injury, $\mathrm{CT}$ is more frequently used than endoscopy because it is less invasive. High-resolution MDCT is useful for evaluating the depth of mucosal damage [9]. Endoscopic dilatation for esophageal stenosis is recommended at least 4 weeks after the acute phase and when there is clear evidence of stricture formation [10]. However, compared with other benign diseases, stenosis due to chemical injury has a high risk of perforation; dilatation must therefore be done carefully, and its success rate is as low as $50 \%[11]$. In the present case, it was inferred that the stomach tissue used for reconstruction had also been damaged by the chemical injury, with no improvement even after frequent dilatation and a subsequent PMMC flap.

During the last 20 years, virtual CT imaging has improved remarkably and continues to be used to address new challenges. For reconstructive surgeons, the greatest advantage of $\mathrm{VE}$ is that it is a noninvasive modality capable of visualizing areas inaccessible to a flexible endoscope. Using the VE findings, we were able to visualize the 3-dimensional shape beyond the stenosis. In addition, VE helped to predict the area of the defect after contracture release. While VE has several advantages, some limitations and pitfalls of VE have been reported. VE lacks the realtime capability of endoscopy, and it is prone to respiratory variations; airway patency may appear falsely compromised compared with the actual data [12]. Lower threshold values may falsely increase the apparent extent of stenosis, and, conversely, higher thresholds may erroneously indicate a mucosal gap [13].

On preoperative imaging in the present case, the stenosis in the vicinity of the piriform sinus was severe and extended for $2-3 \mathrm{~cm}$. In general, free jejunal (tube) transfer is often chosen. However, in this case, there was a risk of damaging the recurrent nerve due to the effects of multiple surgical operations in the past. Compared with a whole jejunal segment, a patch graft is considered to pose a lower risk of nerve injury due to its narrower peeling range. Based on the VE findings, we selected a patch graft, for which we expected that the luminal mucosa could be used to some extent. Thus, a patch graft was chosen based on the VE findings.

Conventionally, a FJPG is transferred in an isoperistaltic fashion, and pooling of food is thus occasionally seen because of the peristaltic contraction and circular folds of the jejunum. Okazaki et al. [8] reported that a $90^{\circ}$-transposed FJPG could overcome this disadvantage. On a videofluorographic study, pooling of 
contrast medium was not seen on postoperative day 10, and oral intake was achieved 6 days earlier than with a conventional FJPG. The patient complained of no discomfort associated with the use of a $90^{\circ}$-transposed FJPG. A limitation of FJPG transfer is that it is not indicated for defects extending to the supraglottic region because of its minimal plasticity [14].

Barrett's esophagus and cancer have been reported to occur an average of 41 years after chemical damage [15], indicating that long-term follow-up is necessary.

\section{NOTES}

\section{Conflict of interest}

No potential conflict of interest relevant to this article was reported.

\section{Ethical approval}

The study was performed in accordance with the principles of the Declaration of Helsinki. Written informed consent was obtained.

\section{Patient content}

The patient provided written informed consent for the publication and the use of his images.

\section{Author contribution}

Concept and design of the study: Fujisawa D. Clinical study: Fujisawa D, Kojya S. Supervised: Asato H. Acquition of the data: Tanaka K, Itokazu T, Fujisawa D. Drafting and revision of the manuscript: Fujisawa D.

\section{ORCID}

Daisuke Fujisawa http://orcid.org/0000-0003-0729-5422

\section{REFERENCES}

1. Vining DJ, Liu K, Choplin RH, et al. Virtual bronchoscopy. Relationships of virtual reality endobronchial simulations to actual bronchoscopic findings. Chest 1996;109:549-53.

2. Lam WW, Tam PK, Chan FL, et al. Esophageal atresia and tracheal stenosis: use of three-dimensional CT and virtual bronchoscopy in neonates, infants, and children. AJR Am J Roentgenol 2000;174:1009-12.

3. Mazzeo S, Caramella D, Gennai A, et al. Multidetector CT and virtual endoscopy in the evaluation of the esophagus. Abdom Imaging 2004;29:2-8.

4. Panebianco V, Grazhdani H, Iafrate F, et al. 3D CT protocol in the assessment of the esophageal neoplastic lesions: can it improve TNM staging? Eur Radiol 2006;16:414-21.

5. Seidenberg B, Rosenak SS, Hurwitt ES, et al. Immediate reconstruction of the cervical esophagus by a revascularized isolated jejunal segment. Ann Surg 1959;149:162-71.

6. Buckspan GS, Newton ED, Franklin JD, et al. Split jejunal free-tissue transfer in oropharyngoesophageal reconstruction. Plast Reconstr Surg 1986;77:717-28.

7. Nakatsuka T, Harii K, Ueda K, et al. Preservation of the larynx after resection of a carcinoma of the posterior wall of the hypopharynx: versatility of a free flap patch graft. Head Neck 1997; 19:137-42.

8. Okazaki M, Asato H, Sarukawa S, et al. Ninety-degree transposed free jejunal patch transfer for hypopharyngeal reconstruction following partial hypopharyngectomy. Plast Reconstr Surg 2008;122:143e-144e.

9. Ryu HH, Jeung KW, Lee BK, et al. Caustic injury: can CT grading system enable prediction of esophageal stricture? Clin Toxicol 2010;48:137-42.

10. Lahoti D, Broor SL, Basu PP, et al. Corrosive esophageal strictures: predictors of response to endoscopic dilation. Gastrointest Endosc 1995;41:196-200.

11. Mowry JB, Spyker DA, Cantilena LR Jr, et al. 2013 Annual Report of the American Association of Poison Control Centers' National Poison Data System (NPDS): 31st Annual Report. Clin Toxicol 2014;52:1032-283.

12. Das KM, Lababidi H, Al Dandan S, et al. Computed tomography virtual bronchoscopy: normal variants, pitfalls, and spectrum of common and rare pathology. Can Assoc Radiol J 2015;66:58-70.

13. De Wever W, Vandecaveye V, Lanciotti S, et al. Multidetector CT-generated virtual bronchoscopy: an illustrated review of the potential clinical indications. Eur Respir J 2004;23:77682.

14. Miyamoto S, Sakuraba M, Asano T, et al. Free jejunal patch graft for reconstruction after partial hypopharyngectomy with laryngeal preservation. Arch Otolaryngol Head Neck Surg 2011;137:181-6.

15. Kim YT, Sung SW, Kim JH. Is it necessary to resect the diseased esophagus in performing reconstruction for corrosive esophageal stricture? Eur J Cardiothorac Surg 2001;20:1-6. 